\title{
La empagliflozina disminuyó la mortalidad en diabéticos tipo 2 de alto riesgo cardiovascular
}

\author{
Empagliflozin reduced mortality in type 2 diabetics at high cardiovascular risk
}

\section{Objetivos}

Evaluar la eficacia y seguridad de la empagliflozina con respecto a la morbimortalidad cardiovascular en pacientes diabéticos tipo 2 de alto riesgo cardiovascular.

\section{Diseño, lugar y pacientes}

Ensayo clínico aleatorizado, doble ciego, multicéntrico. Realizado en 590 centros asistenciales distribuidos en 42 países entre 2010 y 2013. Se incluyeron pacientes mayores de 18 años con diabetes mellitus tipo 2 (DM2) sometidos a tratamiento estándar, con un índice de masa corporal $<45 \mathrm{Kg} / \mathrm{m} 2$, clearence de creatinina $>30$ $\mathrm{ml} / \mathrm{min}$ y enfermedad cardiovascular establecida.

\section{Intervención}

Se aleatorizaron 7.020 pacientes, en una relación 1:1:1, a recibir diariamente empagliflozina $10 \mathrm{mg}$, empagliflozina $25 \mathrm{mg}$ o placebo vía oral. Durante las primeras 12 semanas de tratamiento se instó a mantener, sin modificaciones, cualquier tratamiento hipoglucemiante utilizado al inicio del estudio (aunque se permitían realizar ajustes en casos de glucemia basal > $240 \mathrm{mg} / \mathrm{dl}$, o ante situaciones clínicas particulares). A partir de las 12 semanas y en adelante, se estimuló a los investigadores
Zinman B, y col. N Engl J Med 2015;373:2117. a realizar ajustes de cualquier otro tratamiento para la diabetes a discreción para lograr las metas de glucosa deseadas.

\section{Medición de resultados principales}

El resultado principal fue la combinación de muerte cardiovascular (CV), infarto de miocardio no fatal (IAM) y accidente cerebrovascular no fatal (ACV). Como resultado secundario se consideró la sumatoria del resultado primario más la internación por angina inestable. El análisis se realizó por intención de tratar.

\section{Resultados principales}

Los resultados de los dos grupos asignados a empagliflozina (en dosis diferentes) se analizaron conjuntamente, ya que no se hallaron diferencias significativas entre los mismos (ver tabla 1). El $97 \%$ de los participantes completaron el estudio. El tiempo de observación media fue de 3,1 años. Las diferencias logradas en los valores de hemoglobina glicosilada ( $\mathrm{HbA1C})$ entre los grupos durante el seguimiento se expresan en la tabla 2. Los pacientes asignados a empagliflozina presentaron mayor número de infecciones urinarias y genitales que los asignados a placebo.

Tabla 1. efectos de la empagliflozina respecto de la ocurrencia de eventos cardiovasculares.

\begin{tabular}{l|c|c|c}
\multicolumn{1}{c|}{ Evento } & $\begin{array}{c}\text { Placebo } \\
\mathbf{N = 2 . 3 3 3}\end{array}$ & $\begin{array}{c}\text { Empaglifozina } \\
\text { N=4.687 }\end{array}$ & $\begin{array}{c}\text { Hazard Ratio } \\
\text { (IC 95\%) }\end{array}$ \\
\hline Muerte CV + IAM + ACV & $12,1 \%$ & $10,5 \%$ & $0,86(0,74 \mathrm{a} \mathrm{0,99)}$ \\
\hline Muerte por todas las causas & $8,3 \%$ & $5,7 \%$ & $0,68(0,57 \mathrm{a} 0,82)$ \\
\hline Muerte de causa cardiovascular & $5,9 \%$ & $3,7 \%$ & $0,62(0,49 \mathrm{a} 0,77)$ \\
\hline Hospitalización por insuficiencia cardiaca & $4,1 \%$ & $2,7 \%$ & $0,65(0,50 \mathrm{a} 0,85)$ \\
\hline
\end{tabular}

CV: cardiovascular. IAM: infarto agudo de miocardio. ACV: accidente cerebro vascular.

Tabla 2. diferencia en puntos porcentuales de hemoglobina glicosilada (IC 95\%) según grupo de tratamiento.

\begin{tabular}{l|c|c|c} 
& \multicolumn{2}{|c|}{ Semana } & $\mathbf{9}$ \\
\cline { 2 - 4 } & $\mathbf{1 2}$ & $-0,42(-0,48 \mathrm{a}-0,36)$ & $-0,24(-0,40 \mathrm{a}-0,08)$ \\
\hline Emplagliflozina 10mg & $-0,54(-0,58 \mathrm{a} 0,49)$ & $-0,47(-0,54 \mathrm{a}-0,41)$ & $-0,36(-0,51 \mathrm{a}-0,20)$ \\
\hline
\end{tabular}

\section{Conclusiones}

En pacientes con DM2 de alto riesgo cardiovascular sometidos a tratamiento farmacológico estándar el agregado de empagliflozina redujo la suma de mortalidad CV, IAM y ACV, así como la mortalidad por todas las causas.

Fuente de Financiamiento: laboratorios Boehringer Ingelheim y Eli Lilly.

\section{Comentario}

Los bloqueantes del cotransporte sodio-glucosa 2 (SGLT2), previenen la reabsorción tubular renal de glucosa y sodio, generando glucosuria, y descendiendo a su vez las glucemias plasmáticas. El receptor SGLT2 reabsorbe el $90 \%$ de la glucosa a nivel renal y es un receptor renal específico, en contrapartida del SGLT1, que se encuentra ampliamente expresado en diversos tejidos. A la fecha existen aprobados tres bloqueantes SGLT2 (canagliflozina, dapagliflozina y empagliflozina) por las agencias regulatorias internacionales. En modelos experimentales el animal diabético no tratado tiene aumento de la reabsorción proximal de glucosa y sodio. Esto genera menor concentración de sodio en el nefron distal y el aparato yuxtaglomerular, lo que es censado como hipovolemia, aumentando reactivamente la presión arterial. Los bloqueantes SGLT2 al revertir este proceso modifican la hemodinámica renal y descienden la presión arterial. La reducción de la glucemia y la $\mathrm{HbA}_{1 \mathrm{C}}$ fue en el mejor de los casos modesta en este ensayo. De hecho en la mejor semana llega a -0,5, no explicando por sí sola los contundentes resultados cardiovasculares y de morta- lidad. Se entiende que la reducción del riesgo ha sido multifactorial, no solo por la reducción glucémica, sino también por la modificación de la hemodinámica renal, el descenso de presión arterial, de peso, ácido úrico y albuminuria. Todos estos efectos han sido demostrados en ensayos preclínicos de la droga'.

\section{Conclusiones del comentador}

Este es el primer ensayo clínico en el cual un hipoglucemiante produce disminución de la mortalidad. No obstante persisten algunas dudas e intrigas con respecto a la droga: 1) ¿Cuál es el verdadero lugar de la empagliflozina dentro del diverso y complicado arsenal terapéutico que posee hoy en día la diabetes? 2) ¿Cuál es el balance riesgo beneficio de la droga en pacientes diabéticos sin enfermedad cardiovascular establecida? Sin dudas a su esclarecimiento contribuirán dos ensayos clínicos en curso, el CANAVAS2 y el DECLARE-TIMI583, que evaluarán la canagliflozina y la dapagliflozina en un futuro próximo. 
Lucas Fernández Otero [ Servicio Clínica Médica del Hospital Italiano de Buenos Aires lucas.fernandez@hospitalitaliano.org.ar ]

Fernandez Otero L. La Empagliflozina disminuyó la mortalidad en diabéticos tipo 2 de alto riesgo cardiovascular. Evid Act Pract Ambul. 2016;19(1):15-16. EneMar. Comentado de: Zinman B, y col. Empagliflozin, Cardiovascular Outcomes, and Mortality in Type 2 Diabetes. NEJM. 2015;373(22):2117-28. PMID: 26378978.

Referencias:

1. Abdul Ghani MA. Renal sodium-glucose cotransporter inhibition in the management of type diabetes mellius. Am J Physiol Renal Physiol. 2015 Dec 1;309(11):F889-900. doi: 10.1152/ajprenal.00267.2015. Epub 2015 Sep 9.

2. ClinicalTrials.gov. CANVAS - CANagliflozin cardioVascular Assessment Study (CANVAS) (sitio web). U.S. National Institutes of Health; 01 marzo 2016. Disponible en URL https://clinicaltrials.gov/ct2/show/NCT01032629 (ultimo acceso marzo 2016).

3. ClinicalTrials.gov. CANVAS - Multicenter Trial to Evaluate the Effect of Dapagliflozin on the Incidence of Cardiovascular Events (DECLARE-TIMI58) (sitio web). U.S. National Institutes of Health; 01 marzo 2016. Disponible en URL: https://clinicaltrials.gov/ct2/show/NCT01730534 (último acceso marzo 2016).

$\mathcal{P}$ reunirnos con colegas para compartir nuestras ideas, investigar y difundir nuestros conocimientos con otros profesionales. Asi como asesorar al gobierno y a otras instituciones privadas en la confección de guias que implican cambios en las politicas de Salud Pública, como queda demostrado con la participación de miembros de nuestra asociación en la confección de la Guia Nacional para la EPOC y la revisión del certificado de aptitud fisica.

Además significa integrarnos como médicos de familia a otras asociaciones de Medicina Familiar a nivel nacional, y compartir nuestro saber a nivel internacional, difundiendo nuestra práctica en Congresos dentro y fuera del pais.

\section{¡Vos también podes formar parte de esta transformación!}

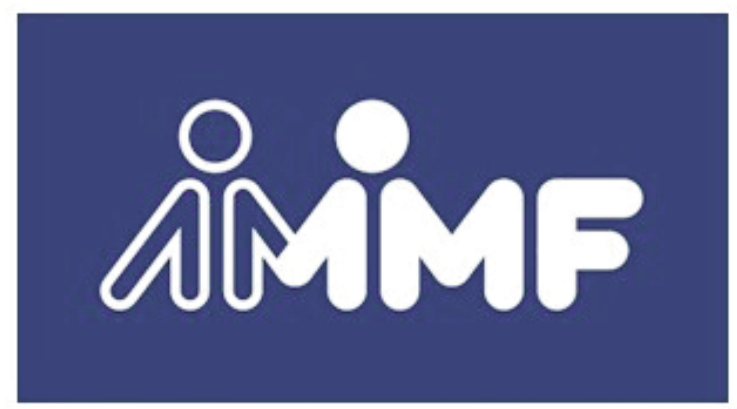

Asociación Metropolitana de Medicina Familiar.

Si queres asociarte o que te brindemos mayor información: secretaria.ammf@gmail.com

Seguinos en Facebook: www.facebook.com/AsociacionMetropolitanadeMedicinaFamiliar 